BNL-113261-2016-JA

\title{
Rejection of Alpha Surface Background in Non-scintillating Bolometric Detectors: The ABSuRD Project
}

\author{
M. Biassoni, C. Brofferio, C. Bucci, L. Canonica, \\ M.L. di Vacri, P. Gorla, M. Pavan, M. Yeh
}

Submitted to Journal of Low Temperature Physics

August 2016

Chemistry Department

Brookhaven National Laboratory

\section{U.S. Department of Energy USDOE Office Of Science, Basic Energy Sciences}




\section{DISCLAIMER}

This report was prepared as an account of work sponsored by an agency of the United States Government. Neither the United States Government nor any agency thereof, nor any of their employees, nor any of their contractors, subcontractors, or their employees, makes any warranty, express or implied, or assumes any legal liability or responsibility for the accuracy, completeness, or any third party's use or the results of such use of any information, apparatus, product, or process disclosed, or represents that its use would not infringe privately owned rights. Reference herein to any specific commercial product, process, or service by trade name, trademark, manufacturer, or otherwise, does not necessarily constitute or imply its endorsement, recommendation, or favoring by the United States Government or any agency thereof or its contractors or subcontractors. The views and opinions of authors expressed herein do not necessarily state or reflect those of the United States Government or any agency thereof. 


\title{
Rejection of alpha surface background in non- scintillating bolometric detectors: the ABSuRD project
}

\author{
M. Biassoni 1 • C. Brofferio 1 • C. Bucci $2 \cdot$ L. Canonica 2 - M.L. \\ di Vacri 2,3 • P. Gorla $2 \cdot$ M. Pavan $1 \bullet$ M. Yeh 4
}

\author{
INFN Sezione Milano Bicocca, MIB, 20126 Milano, Italy 1 \\ INFN Laboratori Nazionali del Gran Sasso, Assergi (AQ), Italy 2 \\ Dipartimento di Scienze Fisiche e Chimiche, Univesità dell'Aquila, \\ L'Aquila, Italy 3 \\ Brookhaven National Laboratory, Upton (NY), USA 4
}

\begin{abstract}
Due to their excellent energy resolution values and the vast choice of possible materials, bolometric detectors are currently widely used in the physics of rare events. A limiting aspect for bolometers rises from their inability to discriminate among radiation types or surface from bulk events. It has been demonstrated that the main limitation to sensitivity for purely bolometric detectors is represented by surface alpha contaminations, causing a continuous background that cannot be discriminated. A new scintillation based technique for the rejection of surface alpha background in nonscintillating bolometric experiments is proposed in this work. The idea is to combine a scintillating and a high sensitivity photon detector with a nonscintillating absorber. We present results showing the possibility to reject events due to alpha decay at or nearby the surface of the crystal.
\end{abstract}

Keywords thermal detectors $\bullet$ scintillation $\bullet$ surface background

\section{Introduction}

A bolometer is a particle detector in which the energy deposited by the incident radiation is converted into phonons and measured based on the temperature change in the detector. It consists of three components: an absorber, a temperature sensor and a weak thermal link connecting the absorber and the sensor to a thermal bath. The temperature variation induced by the interacting particle, which is proportional to the inverse of the heat capacity of the absorber material, is not detectable at room temperature but it becomes measurable by operating the system at cryogenic temperatures. 


\section{Author 1・Author 2 • Author 3}

Temperature variations are of the order of $100 \mu \mathrm{K}$ per MeV. Two advantageous aspects of bolometers have recently made them widely used in experiments searching for rare events [6][7]: the excellent energy resolution that they can achieve (few $\mathrm{keV}$ at $\mathrm{MeV}$ energies) and the vast choice of available absorber materials, restricted only by the specific heat at the operating temperature. A limiting aspect of bolometric detectors is instead represented by their inability to discriminate among radiation types or between surface and bulk events.

Searching for extremely rare events, such as neutrino-less double beta decay or dark matter, implies the removal of any spurious signal that can mimic the searched event. The residual background counting rate limits the sensitivity of experiments. Identification, control and reduction of the radioactive background are thus main issues for the experiments searching for rare events.

A new scintillation based technique for the discrimination of surface alpha background in purely bolometric detectors is described in this work.

\section{The issue of alpha surface background rejection}

The main limitation to sensitivity of purely bolometric experiments of rare events physics is represented by surface degraded alpha contaminations interacting with the absorber [1][2]. An alpha particle generated near the surface of a detector component might release only a part of its energy in the material before escaping and then eventually hit another component of the detector. If one of the two components is non-active, the total energy of the alpha particle cannot be reconstructed; a continuous background is generated at energies below the nominal energy of the alpha particle. Radioactive contaminations close to the absorber surfaces can thus cause signals in the region of energy where the searched event is expected.

The selection of highly radio-pure materials for the experiment assembling, as well as the possibility to identify the particle type interactions ( $\alpha$ from $\beta / \gamma$ ) are fundamental aspects for the reduction and discrimination of the background, and therefore the enhancement of the sensitivity.

The ABSuRD project aims at developing a new scintillation based technique for the rejection of the background generated by surface alpha contaminations in non-scintillating bolometric experiments. The idea is to embed the non-active components of the detector (i.e. holders, clamps or frames) with a scintillating foil and equip the system with a bolometric light detector. The discrimination of the continuous background generated by alpha contaminations becomes possible from the analysis of coincidence signals of heat and light, in the thermal bolometer and in the light detector respectively. A schematic representation of the ABSuRD configuration is in 
Title

Fig. 1 [3]. The advantage of this idea is that it can be applied to non scintillating crystals (e.g. $\mathrm{TeO}_{2}$ ) improving the background rejection capability.

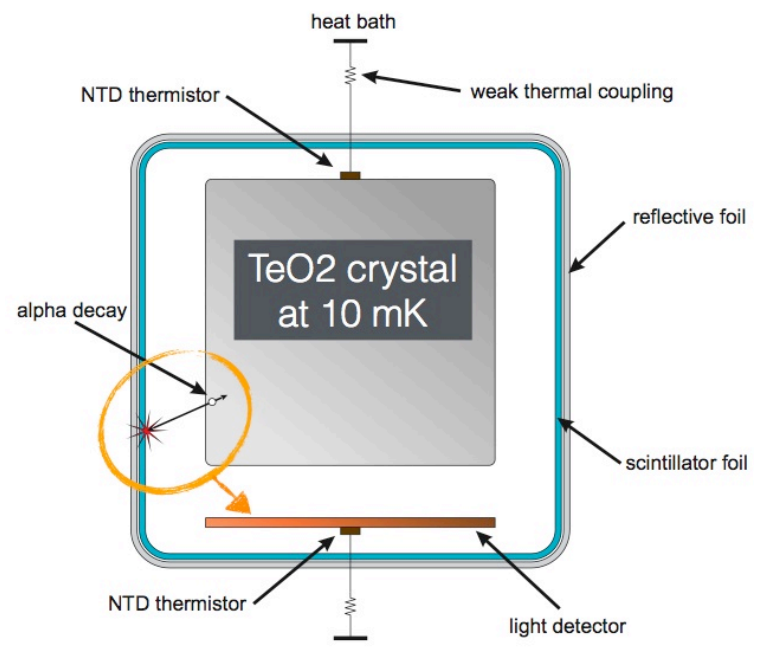

Fig. 1 Schematic representation of the ABSuRD experimental configuration.

\section{Scintillating foils}

Low energy thresholds $(<1 \mathrm{keV})$ are needed for bolometric light detector to be able to detect small light signals produced by alpha contaminations. An alpha particle generated by the decay of ${ }^{210} \mathrm{Po}$, for example, has energy of 5.3 $\mathrm{MeV}$ and produces about $10 \mathrm{keV}$ of photons in commercial scintillating foils at room temperature. Plastic scintillators have an extremely non-linear response to alpha particles [5] and the light produced by a degraded alpha particle of $1.5 \mathrm{MeV}$ is about one order of magnitude smaller than the one produced by a $5.3 \mathrm{MeV}$ alpha. The detection of such a small amount of energy is a challenging task for most of the currently used light detectors operating at cryogenic temperatures (10-20 mK) [4].

Increasing the scintillation light yield and improving the collection efficiency, for example superimposing a reflective foil on the scintillating foil, are desirable to detect low energy alpha particles.

Among them, stability from both chemical and mechanical point of view at cryogenic temperatures, optical transparency, high scintillation light yield at low T. A high degree of purity, mainly from the radioactive point of view, is also required in order not to introduce contaminations that increase the background of the experiment. Custom made scintillating foils resulted to be 


\section{Author 1・Author 2 • Author 3}

the best solution for the ABSuRD project. A set of plastic scintillating foil has been produced using the spin coating technique. Due to its mechanical properties, stability, transparency and absence of total internal reflection, a Teflon based polymer, the THV from $3 \mathrm{M}^{\mathrm{TM}}[8]$, was chosen as a substrate for the film production. Based on the chemical compatibility with the THV, scintillation light yield data and easiness of handling, the system $\mathrm{PC} / \mathrm{PPO} /$ bis-MSB [3] was selected to add scintillating properties to the plastic substrate. An example of a THV+PC/PPO/bis-MSB film spinned on a fused silica glass is shown in Fig 2 (left picture).

\section{Foil characterization facility}

A dedicated cryostat has been set up at LNGS for a preliminary light yield characterization at low temperature for the produced scintillating film. It is a Gifford-McMahon type. A picture of the interior of the system is in Fig. 2 (right picture).

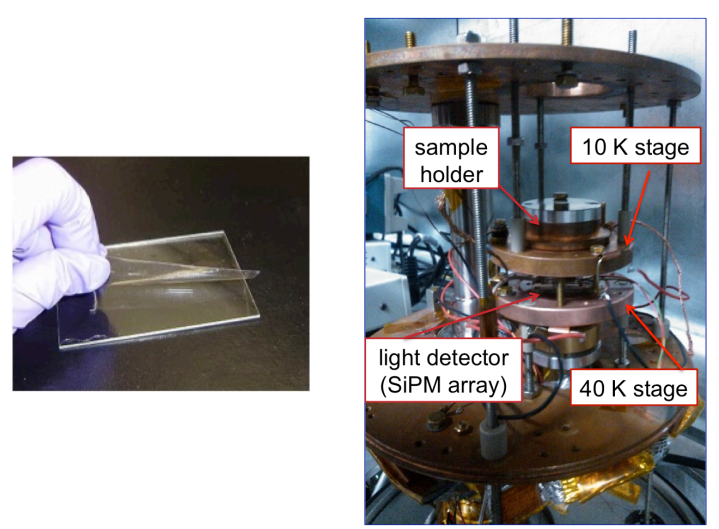

Fig. 2 Example of plastic scintillating foil produced with the spin coating technique (left); Cryostat for the film light yield characterization at low temperature (right).

Two stages can be distinguished (Fig. 2): the $10 \mathrm{~K}$ stage is the one where the sample is located during the measurements. The measurement at this temperature has significance since optical and scintillation properties are not expected to vary in a relevant way below this value. The second stage can be cooled down to $40 \mathrm{~K}$ and is the one where the light detectors are located. An array of 4 Silicon PhotoMultiplier (SiPM) sensors was used. The temperature on the two stages can be independently read and tuned through a temperature controller from Lake Shore, model 335 [9]. The SiPM plate is usually set at $150 \mathrm{~K}$ or above to optimize the operation of these sensors. The 
foil can be irradiated using an alpha source; the cone of scintillation light emitted is detected by the array of SiPM facing the sample. The developed system allows a reliable measurement of the light yield from scintillating samples in a wide range of temperature $(10 \mathrm{~K}-300 \mathrm{~K})$; the characterization of the produced foils is currently on going.

\section{Proof of the ABSuRD technology effectiveness}

The effectiveness of the ABSuRD technology has been verified through a bolometric test. A $3 * 3 * 6 \mathrm{~cm}^{3} \mathrm{TeO}_{2}$ crystal has been operated for 215 hours at $10 \mathrm{mK}$ in a dilution refrigerator at the underground facility of the Gran Sasso National Laboratory (LNGS). The crystal was embedded with a commercial scintillating foil from Saint Gobain (BC400) [10] and a reflective foil from 3M (VM2002). A liquid solution of ${ }^{147} \mathrm{Sm}$ was deposited on one of the crystal surface as an $\alpha$ source (Q-value $2.3 \mathrm{MeV}$ ). The absorber was provided with a Neutron Trasmutation Doped (NTD) Ge thermistor to convert the phonon signal into a detectable voltage pulse. The crystal was placed into a structure of Oxygen Free High Conductivity (OFHC) copper frame (mechanical support and heat sink) and held by PTFE clamps. The top surface of the crystal was faced to a germanium bolometric light detector, equipped with an NTD thermistor as well [4].

The coincidence analysis of the heat and light signals in a defined time window of $50 \mathrm{~ms}$ has demonstrated that this technique is able to tag alpha surface events in the absorber, as shown in the plots of Fig. 3. The energies where the coincidence events in the bolometric detector are concentrated are compatible with nuclear recoil energies of the ${ }^{147} \mathrm{Sm}$ decay $(\sim 80 \mathrm{keV})$. The energies of the corresponding coincidence events detected on the light detector are compatible with the energy associated to the scintillation light produced by an alpha particle emitted in the ${ }^{147} \mathrm{Sm}$ decay.
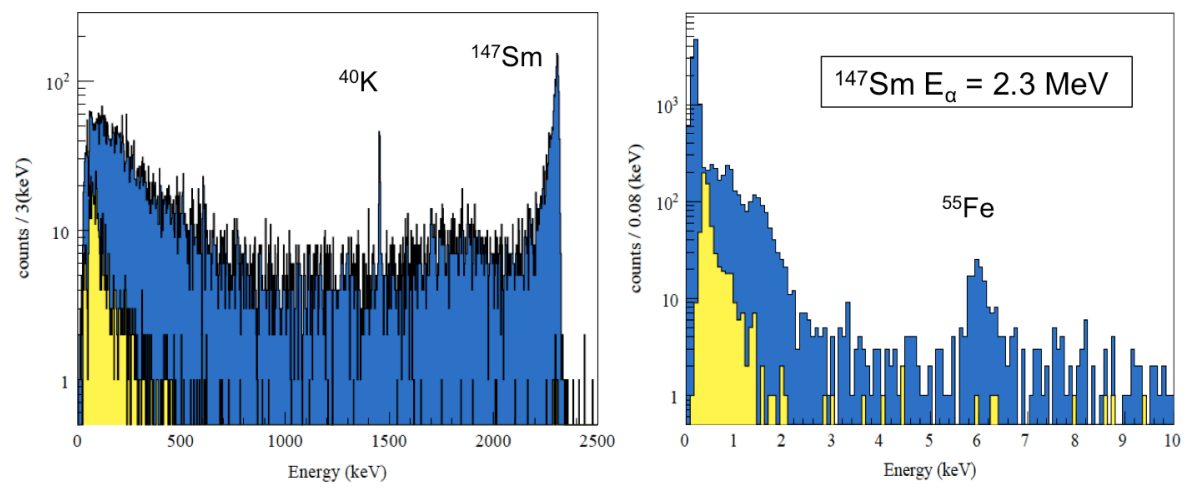


\section{Author 1・Author 2・Author 3}

Fig. 3 Left plot: energy spectrum of the bolometric detector; the events occurring in coincidence with an event on the light detector are the yellow ones, they are overlapped to the total count rate of the crystal. Right plot: energy spectrum of the light detector; the events occurring in coincidence with an event in the absorber are the yellow ones, they are overlapped to the total count rate of the detector.

The test has also confirmed that a scintillating foil with a high light yield in response to alpha particles is required. The response of the BC400 to alpha particles is close to the energy threshold of the used light detector $(\sim 300 \mathrm{eV})$ [10]; an improvement in the efficiency of the technique would be obtained maximizing the light yield of the scintillating foil in response to alpha particles. (More details about the experimental set up, data acquisition and analysis can be found in reference [4]).

\section{Conclusions}

In this work we report the results obtained in the application of a new scintillation based technique for the discrimination of surface alpha background in non-scintillating bolometric experiments searching for rare events. The basic idea of the technique is to develop a hybrid bolometric detector by embedding a non-scintillating bolometer with a scintillating foil and equipping the system with a phonon and a light detector. The discrimination of the background is obtained from a coincidence analysis of the two sensors. The effectiveness of this technique has been proved through a bolometric test using a $\mathrm{TeO}_{2}$ crystal as absorber and a commercial scintillating foil. Since a high scintillation light yield in response to alpha particle and other specific properties, mainly related to the temperature of operation $(\mathrm{mK})$, are required to the foil, a set of custom foil has been produced for the purpose selecting the best polymeric substrate and scintillating system. A dedicated cryostat has been set up for the characterization of the produced foils at $10 \mathrm{~K}$ (below this temperature the scintillation properties are supposed not to vary significantly).

The optimization of the light detector and of the scintillating foil light yield will allow the application of this technology to a low background prototype. The use of the ABSuRD technology in the next generation of purely bolometric experiments searching for rare events will allow the reduction and discrimination of the surface alpha background.

Acknowledgements The authors would like to thank G. Korga, A. Razeto and D. Sablone for useful discussions and help in the installation of light 


\section{Title}

detectors, S. Pirro for providing light detectors and M. Guetti for technical support.

\section{References}

1. C. Arnaboldi et al., Phys Rev C 78, (2008), DOI: 10.1103/PhysRevC.78.035502

2. D. R. Artusa et al., Eur. Phys. J. C (2014) 74:2956

3. J. B. Birks, Pergamon Press (1967)

4. L. Canonica et al., Nucl. Instrum. and Meth. in Physics Research A 732, 286-289 (2013)

5. S.K. Saraf et al., Nucl. Instrum. and Meth. In Physics Research A 138, 451-454 (1990)

6. D. R. Artusa et al., Eur. Phys. J. C (2014) 74:3096

7. L. Cardani et al., J. of Phys.: Conf. Series 375 (2012) 042016

8. http://catalogue.3m.eu

9. www.lakeshore.com

10. www.crystals.saint-gobain.com 